\title{
Dissipation in a quantum wire: fact and fantasy
} $\ddagger$

\author{
Mukunda P. Das ${ }^{1}$ and Frederick Green ${ }^{2}$ \\ ${ }^{1}$ Department of Theoretical Physics, IAS, The Australian National University, \\ Canberra, ACT 0200, Australia. \\ 2 School of Physics, The University of New South Wales, Sydney, NSW 2052, \\ Australia.
}

\begin{abstract}
Where, and how, does energy dissipation of electrical energy take place in a ballistic wire? Fully two decades after the advent of the transmissive phenomenology of electrical conductance, this deceptively simple query remains unanswered. We revisit the quantum kinetic basis of dissipation and show its power to give a definitive answer to our query. Dissipation leaves a clear, quantitative trace in the non-equilibrium current noise of a quantum point contact; this signature has already been observed in the laboratory. We then highlight the current state of accepted understandings in the light of well-known yet seemingly contradictory measurements. The physics of mesoscopic transport rests not in coherent carrier transmission through a perfect and dissipationless metallic channel, but explicitly in their dissipative inelastic scattering at the wire's interfaces and adjacent macroscopic leads.
\end{abstract}

\section{State of Play}

In recent years the understanding of mesoscopic transport has been rewritten through the insights of Landauer, Büttiker, Imry, and others [1, 2]. Succinct and successful, this phenomenology of electron-wave transmission characterizes mesoscopic current flow in terms of two effects: the mismatch of carrier density between large metallic reservoirs (the terminals) across which a low-dimensional conductor (the quantum point contact) of actual interest is connected; and, induced by the density mismatch, lossless quantum transmission of single carriers through the conductor (visualized as a potential barrier).

We are confronted with extremely small structures, possibly of molecular size. Thus they experience a high degree of openness to their macroscopic environment. A striking signature of transport in such a quantum point contact is the discretization of its conductance into "Landauer steps" in units of $2 e^{2} / h \approx 0.078 \mathrm{mS}$.

Landauer's conductance steps are explained via collisionless single-electron quantum transmission through a one-dimensional barrier. The process is evidently elastic; that is, loss-free. However, simple quantum-coherent carrier transmission cannot $\ddagger$ See AIP Conference Proceedings, vol. 1063, pp 26-34 (2008). 
engage with the central issue of conduction: What causes dissipation in a ballistic quantum point contact?

The question is far more than academic, and finding the right answer to it is far more than an esoteric quest. For, in the very near future - if not right now - reliable and effective nano-electronic design will demand theoretical treatments that are not, at their best, merely plausible or cosmetic but credible physically and applicable practically.

It is beyond the scope of coherence-based phenomenologies to cover the necessary physics in a substantial sense. The reason is simple: in their very constitution, coherence methods are incapable of characterizing inelasticity and energy dissipation. So it is not surprising to see, in several classic texts that expound coherent-transmission theory [1, 2, 3], little serious attempt to understand, and come to grips with, the explicit microscopic action of dissipation.

Without a cogent description of dissipation, resistive current flow makes no sense. From the beginning, rather than from afterthought, an answer has to be sought in the manifest physical role of inelastic scattering, and ballistic devices are no exception.

Such a description will connect with first principles to provide a natural explanation for mesoscopic conductance quantization, and much more besides.

In the first part of this paper we outline the answer to our question within the established forms of many-body quantum kinetics [4]. The microscopically based use of many-body methods leads not only to ideal conductance quantization in full account of inelastic energy loss [5], but also resolves a long-standing experimental enigma [6] in the noise spectrum of a quantum point contact (QPC) [7]. Because they follow naturally from microscopics, the same developments foreshadow a systematic pathway to the truly predictive design of novel and useful structures.

In the second part we discuss the idea of the "intrinsic" resistance proper to a quantum wire, and how this important issue is understood from the standpoint of quantum kinetic theory. This topic is all the more necessary to address because there exist well canvassed experimental results that appear to defy the basic microscopic understanding of the intrinsic mesoscopic resistance.

\section{The Physical Issue}

The core issue in the physics of conduction is plain to set out. Any finite conductance $G$ must dissipate electrical energy at the rate $P=I V=G V^{2}$, where $I=G V$ is the current and $V$ the potential difference across the terminals of the driven conductor. It follows that there must be an explicit physical mechanism (emission of optical phonons is one example) by which the net energy gained by carriers, when transported from source to drain, is transferred irreversibly to the surroundings. Alongside any elastic and coherent scattering processes, inelastic processes must always be in place. When these are harnessed together $G$ is uniquely determined; yet it is only the energy-dissipating mechanisms that secure the thermodynamic stability of steady-state conduction.

Since the early work of Callen and Welton, Kubo and later P. C. Martin in the 
'fifties, there has been a complete microscopic understanding of the universal power-loss formula $P=G V^{2}$ (see for example Refs. [8] and [9]). It resides in the fluctuationdissipation theorem, valid for all resistive devices at all scales, in all circumstances. The theorem expresses the requirement for thermodynamic stability. With it comes the conclusion that [4, 5]

- inelasticity is necessary and sufficient to stabilize current flow at finite conductance;

- ballistic quantum point contacts have finite $G \propto 2 e^{2} / h$; therefore

- the physics of energy loss is indispensable to a proper theory of ballistic transport.

The physics of explicit inelastic scattering is beyond the scope of transport models that rely only on coherent quantum scattering to explain the origin of $G$ in quantum point contacts. Coherence implies elasticity, and elastic scattering is always loss-free: it conserves the energy of the scattered particle. This reveals the deficiency of purely elastic models of transmission. We now review a well-defined microscopic remedy for this deficiency.

\section{The Physical Solution}

To allow for the energy dissipation vital to any microscopic description of ballistic transport, we recall that open-boundary conditions imply the intimate coupling of the QPC channel to its interfaces with the reservoirs. The interface regions must be treated as an integral part of the device model. They are the very sites for strong scattering effects: dissipative many-body events as the current enters and leaves the ballistic channel, and elastic one-body events as the carriers interact with background impurities, the potential barriers that confine and funnel the current, and so on.

The key idea in our treatment is to subsume the interfaces within the total kinetic description of the ballistic channel. At the same time, strict charge conservation in an open device requires the direct supply and removal of current by an external generator [9]. Thus the current cannot depend on the physics of the local reservoirs. This canonical requirement sets the quantum kinetic approach entirely apart from Landauerlike treatments [1], which rest upon the phenomenological notion that the current depends on density differences between reservoirs.

\subsection{Ballistic Conductance}

It is straightforward to write the algebra for the conductance in our model system. (For a more detailed description see Ref. 44.) A uniform, one-dimensional ballistic QPC, of operational length $L$, will be associated with two mean free paths determined by $v_{\mathrm{F}}$, the Fermi velocity of the electrons, and a pair of characteristic scattering times $\tau_{\text {el }}, \tau_{\text {in }}$. Thus

$$
\lambda_{\mathrm{el}}=v_{\mathrm{F}} \tau_{\mathrm{el}} ; \quad \lambda_{\text {in }}=v_{\mathrm{F}} \tau_{\text {in }} .
$$




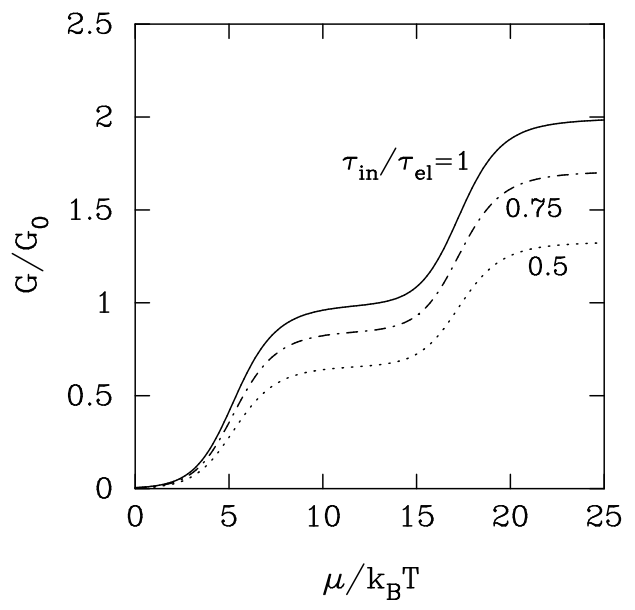

Figure 1. Conductance quantization in a two-band ballistic point contact, as a function of chemical potential $\mu$, calculated with our kinetic theory 4. Full curve: ideal ballistic channels. Broken curves: non-ideal behaviour increases with the onset of inelastic phonon emission inside the contact.

Respectively, these are the scattering lengths set by the elastic and inelastic processes active at both interfaces. From the viewpoint of measurable scattering behaviour, each length encodes the same information as its time.

The device (the QPC and its interfaces) has a conductive core that is collisionless. It follows that

$$
\lambda_{\mathrm{el}}=L=\lambda_{\mathrm{in}} .
$$

Finally, the channel's conductance is given by the familiar formula

$$
G=\frac{n e^{2} \tau_{\text {tot }}}{m^{*} L}=\frac{2 k_{\mathrm{F}}}{\pi} \frac{e^{2}}{m^{*} L}\left(\frac{\tau_{\text {in }} \tau_{\mathrm{el}}}{\tau_{\mathrm{el}}+\tau_{\text {in }}}\right) ;
$$

the effective mass of the carriers is $m^{*}$. In the first factor of the rightmost expression for $G$ we rewrite the density $n$ in terms of the Fermi momentum $k_{\mathrm{F}}$; in the final factor, we use Matthiessen's rule $\tau_{\text {tot }}^{-1}=\tau_{\text {el }}^{-1}+\tau_{\text {in }}^{-1}$ for the total scattering rate in the system.

On making use of equations (11)-(3), the conductance reduces to

$$
G=2 \frac{e^{2}}{\pi \hbar} \frac{\hbar k_{\mathrm{F}}}{m^{*} L}\left(\frac{\left(L / v_{\mathrm{F}}\right)^{2}}{2 L / v_{\mathrm{F}}}\right)=\frac{2 e^{2}}{h} \equiv G_{0} .
$$

This is exactly the Landauer conductance of a single, one-dimensional, ideal channel.

In Figure 1 we plot the results of our model for a QPC [4] made up of two onedimensional conduction sub-bands with threshold energies energies set at $5 k_{\mathrm{B}} T$ and $17 k_{\mathrm{B}} T$, in thermal Boltzmann units $k_{\mathrm{B}} T$. We have used the natural extension of equation (4) to the case where more than one channel may open up to conduction, depending on temperature $T$ and the size of the chemical potential $\mu$. As we increase the role of inelastic scattering by making $\tau_{\text {in }}$ shorter than $\tau_{\text {el }}$ the conductance duly undershoots its ideal, ballistic Landauer limit. But its step structure survives. 

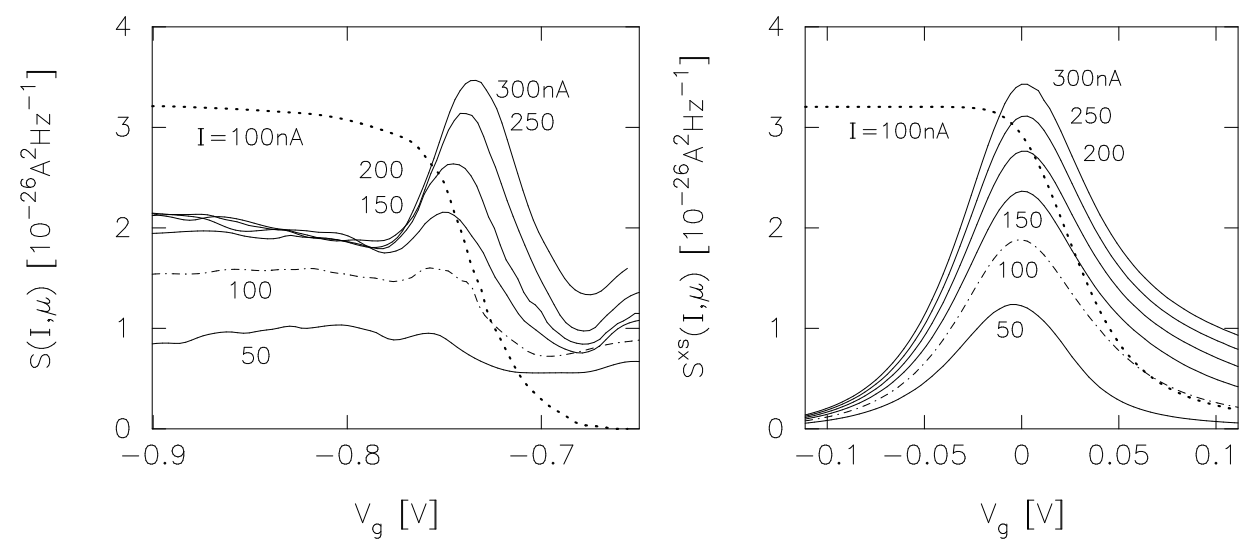

Figure 2. Non-equilibrium current noise of a QPC at constant source-drain current, as a function of gate bias. Left: data from Reznikov et al. [6]. Right: calculation from Green et al. [7]. In each case the dotted line traces the standard shot-noise prediction at 100nA using, as respective inputs, measured and calculated data for $G$. The standard prediction is well wide of the mark.

None of the singular assumptions, commonly adduced to explain conductance quantization as coherent transmission [1, 2], is needed by our conserving microscopic approach. Indeed, the result emerges naturally from completely standard quantum kinetics.

What is the crux of the logic of equation (4)? It is precisely the manifest and indispensable role of inelastic energy loss, acting in concert with elastic scattering and on a completely equal physical footing. That its action is one of the underpinnings of quantum transport is expressed in the fluctuation-dissipation theorem [8].

Charge conservation - the other and concurrent underpinning - is always guaranteed if one applies proper microscopically consistent open-boundary conditions at the interfaces [4, 5, 9]. These twin canonical requirements, imposed by physics and not by anything else, have so far not been evident within heavily phenomenological derivations of equation (44). It remains a challenge for the alternative phenomenologies of ballistic conductance to demonstrate their direct provenance from microscopics, at least to the same level of logical consistency as the quantum kinetic analysis.

\subsection{Non-equilibrium Noise}

The noise response of a quantum point contact is a fascinating aspect of mesoscopic transport, and a more demanding one both experimentally and theoretically. It is in the noise characteristics of a ballistic wire that the predictive capacity of a quantum kinetic description comes really to the fore.

In 1995, a landmark measurement of non-equilibrium noise was performed by the Weizmann group [6], which yielded a very puzzling result. Whereas conventional models 
[2] predicted a strictly monotonic shot-noise signal for a QPC driven at constant current levels, the data showed a series of very strong peaks at threshold (where the carrier density in the QPC rapidly grows and becomes metallic). This is in marked contradiction to theoretical expectations.

Remarkable as they still are, the Weizmann results remained absolutely unexplained for a decade. We have now accounted for them within our strictly conserving kinetic description [7].

In Fig. 2 we display the experimental data side by side with our computation of excess QPC noise under the same conditions [7]. There is close accord between the measured constant-current peaks and our calculation. This is in obvious contrast to standard phenomenology [2] which predicts no peaks whatsoever in this situation.

It is worth noting that the additional measurements of noise-peak structures carried out at constant source-drain voltage [6] (a more common experimental practice) are as well described by our quantum kinetics as they are by any coherent-transmission model. Furthermore, our calculation [7] also shows a substantial monotonically rising noise background at higher gate voltages. This feature is quantitatively consistent with observations, and again one that is completely absent from phenomenological models of the same data $[2]$.

\section{Intrinsic Resistance of a Quantum Wire: Fact or Fantasy?}

\subsection{Perfect Conductor}

Before analyzing the intrinsic resistance we stress once more the central physical property of the Landauer formula deduced from quantum kinetics: it demands quantitative inclusion of inelastic scattering if it is to emerge at all naturally. In the foregoing discussion of equation (4) we have treated resistance as the total resistance of the mesoscopic device in series with the access resistance of the interfaces and macroscopic leads, the latter being the actual reference contacts for external measurements.

The "intrinsic" resistance of a mesoscopic device should therefore exclude all the access resistances: not only those belonging to the external apparatus but, crucially, those of the boundaries with the leads. We do not have space here to cover fully the quantum kinetic approach to intrinsic resistance. It is the outcome of the system's overall response to an external voltage, by which the field internal to the device is minimized through self-consistent electrostatic screening at the boundaries. A detailed microscopic account may be found, for example, in the study by Kamenev and Kohn [10] who apply standard first-principles methodology [8] to the problem.

A practical way to characterize the intrinsic (in some real sense, actual) device resistance is to attach non-invasive voltage probes across the structure in such a way that local current flow is undisturbed by any measurement of the voltage drop between the probes. The set-up of two current probes at the macroscopic leads, augmented with 
two non-invasive voltage probes as close as possible to the device, is referred to as a "four-terminal" arrangement. Provided one can quantify the degree of disturbance of the current by the inner terminals, this approach gives practical meaning to theoretical discussion of the real resistance of a mesoscopic wire.

A striking example of a four-terminal measurement was given by de Picciotto et al. [11] In this experiment, both two- and four-terminal resistances were measured for an essentially ballistic, one-dimensional conducting channel with inner probes designed to minimally disturb the current in the device.

From two-terminal resistance measurements (without internal probing; device and leads configured in series) de Picciotto et al. obtained strong quantized steps in the resistance, close to the ideal Landauer prediction for a ballistic quantum channel; close but not quite coincident, for the steps all show a clear shortfall of about $7 \%$ from ideal quantization. On the other hand, the intrinsic resistance of the same structure was measured to be vanishingly small.

What is the interpretation of this experiment? First, in its two-terminal version the Landauer formula tells us that, given probability $\mathcal{T}$ for electron-wave transmission through the channel, the source-drain resistance for a single occupied sub-band is

$$
R_{2}=\frac{h}{2 e^{2}} \frac{1}{\mathcal{T}}
$$

In an ideal case $\mathcal{T}=1$. If the measured deviation in $R_{2}$ is a few percent, one can infer a non-ideal $\mathcal{T}<1$. Second, the four-terminal resistance - intrinsic to the device alone - is 12

$$
R_{4}=\frac{h}{2 e^{2}}\left(\frac{1}{\mathcal{T}}-1\right) .
$$

Büttiker [13] has derived a more general four-terminal relation in terms of the partial transmission amplitudes between any one probe and any other. When invasive influences are small, as in actual measurements, this formula can be shown to be equivalent to equation (6) [14].

According to the four-terminal result of Ref. [11], namely that the intrinsic resistance $R_{4}$ vanishes, it follows that $\mathcal{T}$ is unity. There is no intrinsic resistance here. A fortiori there is none that can be said to be quantized.

In another experiment Reilly et al. [15] also measured the four-terminal conductance $\left(1 / R_{4}\right)$ of a ballistic conductor. Their raw data exhibit a perfect Landauer staircase as a function of voltage applied via a side gate to modulate the channel width. For this result Reilly et al. stress that "no attempt has been made to adjust the plateau heights to fit with quantized units of $2 e^{2} / h$." That is: (i) $R_{4}$ is free of artefacts and (ii) the intrinsic conductance is decidedly finite and so is $R_{4}$.

Regarding the Landauer formulae (5) and (6), one immediately faces a difficulty with respect to their universal and mutually consistent understanding. For, in the literature we are being offered two completely different observations of $R_{4}$ (the nominally intrinsic resistance of a clean quasi-one-dimensional quantum wire): in one measurement 
it is strongly quantized as multiple, evidently finite, steps [15] while in the other it essentially vanishes [11] as might be ideally expected.

\subsection{Negative Intrinsic Resistance}

A more recent set of four-point measurements by Gao et al. [16] shows that $R_{4}$ can even be negative. This might be explained by the general Büttiker four-terminal resistance formula [13. If so, however, this would also entail a set of contradictions [17]:

- if $R_{4}<0$ then $\mathcal{T}>1$ and unitarity (probability) is not conserved albeit the Landauer model is predicated on unitary single-electron propagation.

- If $R_{4}<0$ then the power dissipation in the device proper is $P=I^{2} R_{4}<0$ and the device must spontaneously be giving up energy to the rest of the circuit.

The same dilemmas arise for yet another, three-terminal, experiment [18] announcing the measurement of "absolute negative resistance". Their explanation is again in terms of the Büttiker three-terminal formula (a restriction of the four-terminal relation). Nevertheless all the transmission probabilities, being positive, cannot produce a negative resistance from the three-terminal Büttiker formula. Just as before the negative resistance in this experiment directly violates the Büttiker theory.

The above are only a few examples of the counter-intuitive consequences readily drawn from the accepted literature on mesoscopic transport (taking on trust the integrity of works that have been peer reviewed). It is clear that various significant experiments on ballistic conduction are in contradiction not only with prevailing theory but also with one another, if not internally.

Thus it is not unfair to ask how much of the content of certain published experiments is actual fact and how much of it begs certain favoured questions, having more the character of wish fulfilment than hard reality. But such is not for us to analyze; readers are free to form their own conclusions - or not.

\section{Summary}

We have argued that a canonical kinetic approach to mesoscopic transport provides uniquely - a microscopic account of conductance and noise in quantum point contacts. It does so, and will always do so, free of gratuitous guesswork and question-begging.

Unified models of quantum transport and fluctuations will yield, and have yielded already, a natural and detailed understanding of core non-equilibrium processes inside a quantum wire. Fluctuations, by way of current noise, carry much more information on the internal dynamics of mesoscopic systems. Such knowledge is not accessible through the current-voltage characteristics alone, so that further device-noise experiments, especially at constant current, would be of foremost importance.

To our mind, the heart and soul of a mesoscopic theory rest with its physical integrity, and securing its credibility requires sustained, diligent and harmonious work 
by experimentalists and theorists. But the theoretical task is not made any easier when experimental works, appearing in the record, contradict one another and even themselves - not to mention the entire spectrum of mesoscopic models (widely accepted or otherwise).

\section{References}

[1] Y. Imry, Introduction to Mesoscopic Physics, 2nd ed (Oxford University Press, Oxford, 2002).

[2] Y. M. Blanter and M. Büttiker, Phys. Rep. 336, 1 (2000).

[3] P. A. Mello and N. Kumar, Quantum Transport in Mesoscopic Systems; Complexity and Statistical Fluctuations (Oxford University Press, Oxford, 2004).

[4] M. P. Das and F. Green, J. Phys.: Condens. Matter 15, L687 (2003).

[5] F. Green and M. P. Das, J. Phys.: Condens. Matter 12, 5233 (2000); ibid, 5251.

[6] M. Reznikov, M. Heiblum, H. Shtrikman, and D. Mahalu, Phys. Rev. Lett. 75, 3340 (1995).

[7] F. Green, J. S. Thakur, and M. P. Das, Phys. Rev. Lett. 92, 156804 (2004).

[8] R. Kubo, M. Toda, and M. Hashitsume, Statistical Physics II: Nonequilibrium Statistical Mechanics, 2nd ed (Springer, Berlin, 1991).

[9] F. Sols, Phys. Rev. Lett. 67, 2874 (1991); W. Magnus and W. Schoenmaker, Quantum Transport in Sub-micron Devices: A Theoretical Introduction (Springer, Berlin, 2002).

[10] A. Kamenev and W. Kohn, Phys. Rev. B 63, 155304 (2001).

[11] R. de Picciotto, H. L. Stormer, L.N. Pfeiffer, K. W. Baldwin, and K. W. West, Nature 41151 (2001).

[12] H. L. Engquist and P. W. Anderson, Phys. Rev. B 24, 1151 (1981).

[13] M. Büttiker, Phys. Rev. Lett. 57, 1761 (1986).

[14] D. K. Ferry and S. M. Goodnick, Transport in Nanostructures (Cambridge University Press, Cambridge, 1997).

[15] D. J. Reilly et al, Phys. Rev. B 63, 121311(R) (2001).

[16] B. Gao et al, Phys. Rev. Lett. 95, 196802 (2005).

[17] M. P. Das, F. Green, J. S. Thakur, arXiv preprint cond-mat/0601595 (2006).

[18] I. I. Kaya and K. Eberl, Phys. Rev. Lett. 98, 186801 (2007). 